\title{
AN ALMOST UNIVERSAL FORM
}

\section{GORDON PALL}

P. R. Halmos ${ }^{1}$ obtained the 88 possible forms $(a, b, c, d), 0<a \leqq b$ $\leqq c \leqq d$, which represent all positive integers with one exception, and proved that property for all except for the form $h=(1,2,7,13)$. A proof for $h$ follows.

The forms $f=(1,2,7)$ and $g=(1,1,14)$ constitute the reduced forms of a genus. ${ }^{2}$ Between them they represent all positive integers not of the form ${ }^{3} \Lambda=7^{2 k+1}(7 m+3,5,6)$. The identities

$$
\begin{aligned}
x^{2}+y^{2}+14 z^{2} & =x^{2}+2((y+7 z) / 3)^{2}+7((y-2 z) / 3)^{2} \\
& =y^{2}+2((x+7 z) / 3)^{2}+7((x-2 z) / 3)^{2}
\end{aligned}
$$

show that every number represented by $g$ with either $y \equiv-z$ or $x \equiv-z(\bmod 3)$ is also represented by $f$. Hence every number $3 n$ and $3 n+1$ not of the form $\Lambda$ is represented by $f$. For, $x \equiv y \equiv 0, z \neq 0$, and $x, y \neq \equiv, z \equiv 0(\bmod 3)$ both imply $g \equiv 2$. If $N=3 n$ or $3 n+1$ is of the form $\Lambda$, then $7 \mid N$, so that $N-13 \cdot 3^{2} \neq \Lambda$. Similarly, one of $3 n+2-13$ and $3 n+2-52$ is not of the form $\Lambda$; but neither of these is congruent to $2(\bmod 3)$. These linear forms are positive if $n \geqq 39 ; h$ represents all integers not less than 119 . The only number less than 119 not represented in $(1,2,7,13)$ is found to be 5 .

MCGill University

1 This Bulletin, vol. 44 (1938), pp. 141-144.

2 See any table of positive ternaries.

${ }^{3}$ For example, see B. W. Jones, Transactions of this Society, vol. 33 (1931), pp. 111-124. 